\title{
The Isoperimetric Problem for Pinwheel Tilings
}

\author{
Charles Radin ${ }^{1}$, Lorenzo Sadun ${ }^{2}$ \\ Mathematics Department, University of Texas, Austin, TX 78712, USA \\ E-mail: radin@math.utexas.edu and sadun@math.utexas.edu
}

Received: 1 April 1995/in revised form: 9 May 1995

\begin{abstract}
In aperiodic "pinwheel" tilings of the plane there exist unions of tiles with ratio (area)/(perimeter $)^{2}$ arbitrarily close to that of a circle. Such approximate circles can be constructed with arbitrary center and any sufficiently large radius. The existence of such circles follows from the metric on pinwheel space being almost Euclidean at large distances; if $P$ and $Q$ are points separated by large Euclidean distance $R$, then the shortest path along tile edges from $P$ to $Q$ has length $R+o(R)$.
\end{abstract}

\section{Introduction and Statement of Results}

The classic isoperimetric problem in the plane, which asks for the curve of least length enclosing some fixed area, has stimulated much important mathematics. One generalization which has developed within geometric measure theory treats spaces less symmetric than the Euclidean plane, such as spaces representing the structure of crystals. Due to the periodic arrangement of their atoms such structures are, on a macroscopic scale, invariant under translations but not rotations. This has easily observed consequences for crystals; for quartz or table salt one can literally see an optimal polyhedral shape, a shape which solves a version of the isoperimetric problem that can be described as follows. There is a "cost function" $f(\vec{n})$ associated with variable normal directions $\vec{n}$ of planes in space. (Physically, $f(\vec{n})$ is the energy per unit area needed to separate a crystal into two parts along a plane with normal $\vec{n}$.) The problem is to imagine integrating $f$ over each possible surface enclosing a region of fixed volume $V$, and then to find the surface which minimizes this integral. In 1901 Wulff gave a simple construction for such optimal surfaces (see [Wul, Tay]) which is still used to produce the polyhedral "Wulff shapes" for common crystals.

In this paper we generalize this isoperimetric problem to geometries associated with quasicrystals. Quasicrystals are an exotic class of solids, usually metallic alloys, \footnotetext{
13.

${ }^{2}$ Research supported in part by an NSF Mathematical Sciences Postdoctoral Fellowship and Texas ARP Grant 003658-037.
} 


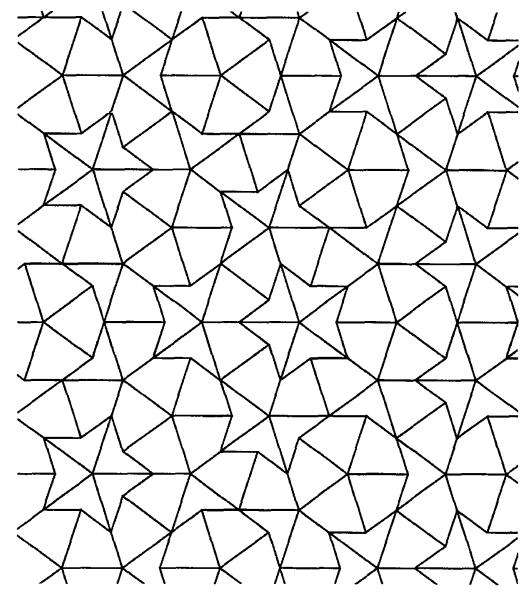

Fig. 1. A Penrose tiling

with an internal structure more complicated than that of ordinary periodic crystals [S-O]. A common technique for modeling such materials utilizes aperiodic tilings of space by polyhedra; the best known examples are three dimensional versions of Penrose's polygonal tilings of the plane [Gar]; Fig. 1. For tilings of the plane there is a natural isoperimetric problem yielding the Wulff shape, as follows: Given a tiling of the plane, find that collection of tiles which covers a fixed area $A$ with boundary of minimal length. In order to obtain the necessary perspective of macroscopic shape compared to atomic substructure, it is appropriate to seek an optimal shape asymptotic in $A \rightarrow \infty$.

There are no real difficulties determining the Wulff shapes for some such structures. For instance, it is not hard to see that the Wulff shape for Penrose tilings is a regular decagon. However, there are natural analogs of the Penrose tilings, exhibiting more unusual symmetries, for which the Wulff shape is not so obvious.

In this paper we consider a certain "pinwheel" tiling of the Euclidean plane made by congruent copies of a $1,2, \sqrt{5}$ right triangle (see Fig. 2), and determine the shape of that region of fixed area which can be covered by a portion of the tiling with least perimeter. (See $[\operatorname{Rad} 2, \operatorname{Rad} 3]$ for discussions of pinwheel tilings and [B-R, Rad1, Rad5] for the relation to possible quasicrystals.) We solve the isoperimetric problem asymptotically for large area, and prove that the optimal limiting shape is a smooth circle, in that there are regions for which area $/(\text { perimeter })^{2}$ is arbitrarily close to $(4 \pi)^{-1}$. In fact we prove that large approximate circles are ubiquitous:

Theorem 1. Given $\varepsilon>0$ there is a distance $R$ such that, for any disk $C$ with radius $r>R$ there is a region $D$, whose boundary follows the edges of triangles in the tiling, that approximates $C$ in the sense that

$$
\mid(\text { perimeter of } D)-2 \pi r \mid<\varepsilon r, \quad(\text { area of } C \backslash D)+(\text { area of } D \backslash C)<\varepsilon r^{2} \text {. }
$$

In particular, $($ area of $D) /(\text { perimeter of } D)^{2}>(4 \pi)^{-1}-\varepsilon$.

This follows from an approximate metric on the space. For large separations, the length of the shortest path between two points $P, Q$ is approximately the Euclidean distance $\|P-Q\|$. More precisely, 


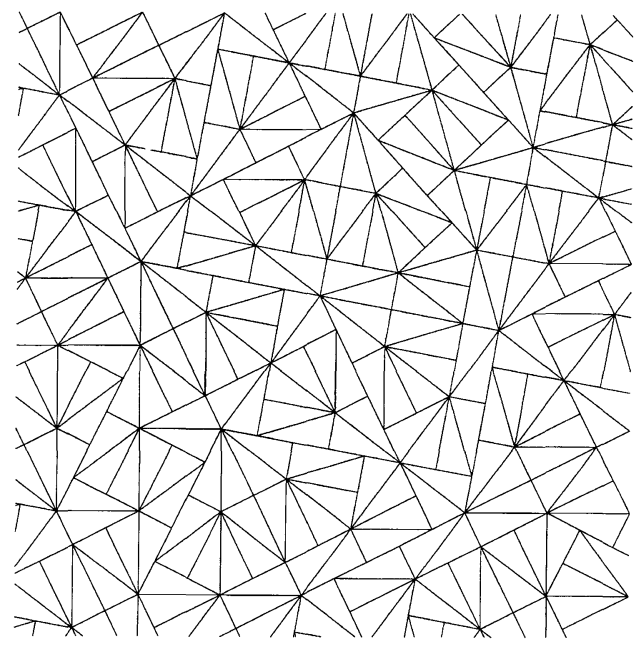

Fig. 2. A pinwheel tiling

Theorem 2. Given any $\varepsilon>0$ there exists $R$ such that, for any two points $P, Q$ on the boundaries of triangles with $\|P-Q\|>R$, there is a path $h$ along the boundaries of these triangles, connecting $P$ to $Q$, with length $|h|$ satisfying

$$
\frac{|h|-\|P-Q\|}{\|P-Q\|}<\varepsilon .
$$

\section{The Details}

The pinwheel tiling is constructed as follows. We start with a $1,2, \sqrt{5}$ right triangle, divide it into five similar triangles as in Fig. 3, and then expand the collection of five triangles by the factor $\sqrt{5}$. We now have five triangles congruent to the original. Move and rotate this collection so that the middle triangle coincides with the triangle we started with. Now subdivide each of the five triangles to produce a collection of twenty-five triangles and again rescale by $\sqrt{5}$. Move and rotate this collection so the middle five triangles, highlighted in Fig. 3, coincide with the five triangles from the previous stage. Repeat this process of subdivision, rescaling and movement ad infinitum, with the central fifth of each new pattern always coinciding with the entire previous pattern. The end result is the pinwheel tiling.

As a first step towards Theorem 2, we prove a similar result about subdividing triangles without expanding or moving. Take the basic $1,2, \sqrt{5}$ right triangle, which we denote $T$, subdivide it into five similar triangles, subdivide each of those triangles, and so on. (See Fig. 4 for the result of 5 subdivisions.) Let $X$ be the space of pairs of points on the boundary of $T$, with the product topology. For any $p=(P, Q) \in X$, let $f(p, n)$ be the minimum distance along a path from $P$ to $Q$ along edges of triangles in the $n^{\text {th }}$ subdivision.

Theorem 3. For any $p=(P, Q) \in X, \lim _{n \rightarrow \infty} f(p, n)=\|P-Q\|$. 


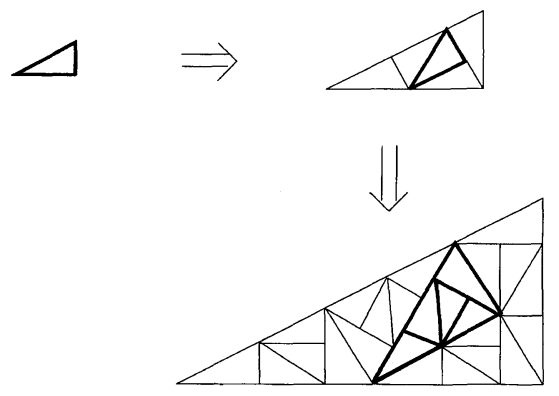

Fig. 3. Two iterations of the pinwheel construction, without rotations

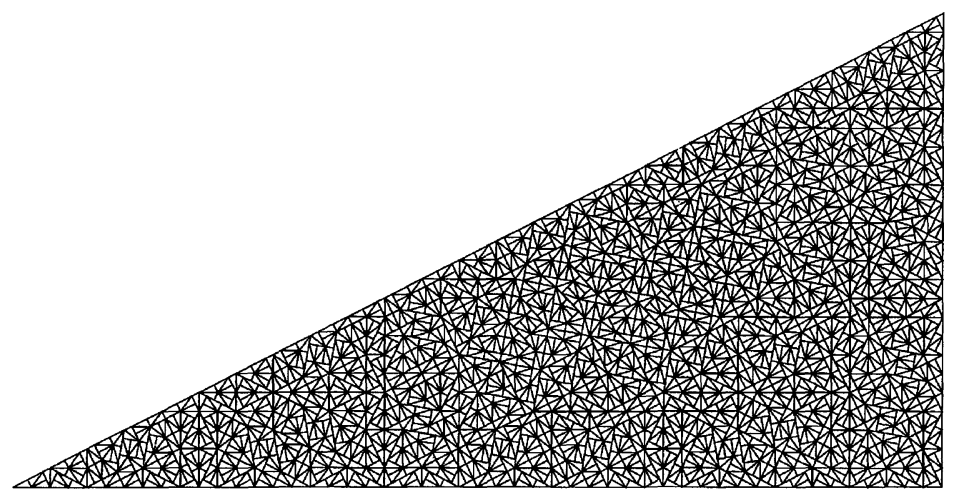

Fig. 4. Result of 5 subdivisions

Proof. When $P=Q$ the result is trivial, so we henceforth only consider pairs of distinct points. Let $g(p, n)=f(p, n) /\|p\|$, where $\|p\|$ is the Euclidean distance between the points in $p$. Let $F(p)=\inf _{n \geqq 0} f(p, n)$ and let $G(p)=\inf _{n \geqq 0} g(p, n)=$ $F(p) /\|p\| . F$ is continuous on $X$, so $G$ is continuous where defined. We first show that $G$ attains its supremum on the compact subset $X^{\prime}=\{p \in X \mid\|p\| \geqq 0.01\}$. We then show that this supremum is 1 . Since $G \geqq 1$, this shows that $G$ is identically 1 , which is tantamount to Theorem 3 .

The points in $X \backslash X^{\prime}$ are of two types. Either both points lie on the same edge, in which case $G(p)=g(p, 0)=1$, or $P$ and $Q$ are close to, but on opposite sides of, one of the three vertices. The theorem clearly holds for the first type, so we consider the second type. Let $p_{n}$ be the pair of points $5^{n}$ times farther from the given vertex than $P$ and $Q$. For pairs near the acute angles, subdividing twice shows that $g(p, n)=g\left(p_{1}, n-2\right)$, and so $G(p)=G\left(p_{1}\right)=G\left(p_{2}\right)$, etc. Eventually one of the pairs $p_{n}$ will lie in $X^{\prime}$, so $G(p) \leqq \sup _{p^{\prime} \in X^{\prime}} G\left(p^{\prime}\right)$. Finally, subdividing once shows that, for pairs near the right angle, $G(p)$ is bounded by a weighted average of $G\left(p^{\prime}\right)$ for pairs $p^{\prime}$ near the two acute angles, and therefore by $\sup _{p^{\prime} \in X^{\prime}} G\left(p^{\prime}\right)$. Thus $\sup _{p \in X} G(p)=\sup _{p \in X^{\prime}} G(p)$.

Note that $\lim _{n \rightarrow \infty} g(p, n-k)=G(p)$ for any finite $k$. This implies that, for any two points $P, Q$ in the $k^{\text {th }}$ subdivision of $T$, there exists an $n$, and a path from 
$P$ to $Q$ along edges of the $n^{\text {th }}$ subdivision, whose length is arbitrarily close to (or less than) $\sup _{p \in X} G(p)\|P-Q\|$.

Since $X^{\prime}$ is compact, $\sup _{p \in X^{\prime}} G(p)=\max _{p \in X^{\prime}} G(p)$. We will prove this equals 1 by contradiction. So assume there is a $\tilde{p} \in X^{\prime}$ such that $\max _{p \in X^{\prime}} G(p)=G(\tilde{p})=$ $1+\delta$ with $\delta>0$. If $\tilde{P}, \tilde{Q}$ are the points in $\tilde{p}$, let $\tilde{H}$ be the straight line joining $\tilde{P}$ to $\tilde{Q}$.

Perform $m$ subdivisions on the original triangle $T$ so that $T$ contains a triangle $T^{\prime}$ intersected by $\tilde{H}$, with this intersection in the middle third of the line. (Further requirements on $m$ will appear later.) Fix $n$ such that for each line determined by a $p \in X$ there is contained in the triangle of level $n$ some triangle of level 0 for which the small edge makes an angle $\theta$ with respect to the line, with $1 / \cos (\theta)<1+\delta / 2$ (this is possible from $[\operatorname{Rad} 4]$ ). Now subdivide the triangle $T$ an additional $n$ times, obtaining a triangle $T^{\prime \prime}$ within $T^{\prime}$ for which the short edge makes an angle of $\theta$ with respect to $\tilde{H}$, with $1 / \cos (\theta)<1+\delta / 2$.

Let $L_{1}$ be the straight line joining $\tilde{P}$ to $t_{1}$, the vertex on the short edge of $T^{\prime \prime}$ closer to $\tilde{P}$, let $L_{2}$ be that short edge of $T^{\prime \prime}$, and let $L_{3}$ be the straight line joining $t_{2}$, the vertex on the short edge of $T^{\prime \prime}$ closer to $\tilde{Q}$, to $\tilde{Q}$. Let $l_{1}$ (resp. $l_{2}$, resp. $l_{3}$ ) be the projection of $L_{1}$ (resp. $L_{2}$, resp. $L_{3}$ ) onto $\tilde{H}$. We denote the length of a line $L$ by $|L|$; in particular, for $L_{2}$, which is a short edge obtained by subdividing $m+n$ times the triangle $T$, we have $\left|L_{2}\right|=5^{-(m+n) / 2}$. Since $T^{\prime}$ was obtained by subdividing $m$ times, it follows that

$$
\left|L_{1}\right| \leqq\left(\left|l_{1}\right|^{2}+5^{1-m}\right)^{1 / 2}=\left|l_{1}\right|\left(1+\frac{5^{1-m}}{\left|l_{1}\right|^{2}}\right)^{1 / 2}<\left|l_{1}\right|+\frac{5^{1-m}}{\left|l_{1}\right|}
$$

So

$$
\left|L_{1}\right|-\left|l_{1}\right|<\frac{5^{1-m}}{\left|l_{1}\right|}<3 \frac{5^{1-m}}{|\tilde{H}|},
$$

since $\left|l_{1}\right|>|\tilde{H}| / 3$. By the same reasoning the same inequality holds for $L_{3}$, namely

$$
\left|L_{3}\right|-\left|l_{3}\right|<3 \frac{5^{1-m}}{|\tilde{H}|} .
$$

Now $\left|L_{2}\right|=\left|l_{2}\right| / \cos (\theta)<\left|l_{2}\right|(1+\delta / 2)<2\left|l_{2}\right|$, so

$$
\left|l_{2}\right|(1+\delta)-\left|L_{2}\right|>\left|l_{2}\right| \frac{\delta}{2}>\left|L_{2}\right| \frac{\delta}{4}=5^{-(n+m) / 2} \frac{\delta}{4}
$$

But, if we choose $m$ such that

$$
5^{-(m+n) / 2} \frac{\delta}{4}>6 \frac{5^{1-m}}{|\tilde{H}|}(1+\delta)
$$

which is certainly possible, then we get a contradiction with the optimality of $\tilde{p}$ by the following argument. By assumption there is a path along the edges of some subdivision, connecting the endpoints of $L_{1}$ (resp. $L_{3}$ ), whose length is arbitrarily close to (or shorter than) $\left|L_{1}\right|(1+\delta)$ (resp. $\left|L_{3}\right|(1+\delta)$ ). Thus there is such a path connecting the endpoints of $\tilde{H}$ whose length is arbitrarily close to (or shorter than) $\left|L_{1}\right|(1+\delta)+\left|L_{2}\right|+\left|L_{3}\right|(1+\delta)$. From (4)-(7) this is less than $|\tilde{H}|(1+\delta)$, which is the contradiction. 
We now return to the pinwheel tiling. We call the $1,2, \sqrt{5}$ triangles that make up the tiling "level-0 triangles". Each such triangle, combined with four of its neighbors, forms a $\sqrt{5}, 2 \sqrt{5}, 5$ "level-1 triangle". Each level-1 triangle, together with four of its neighbors, forms a 5,10,5 $\sqrt{5}$ "level-2 triangle", and so on; see Fig. 5. If $P$ and $Q$ are points on the boundary of a level- $n$ triangle, then the shortest path from $P$ to $Q$ along level-0 triangles has length $\|P-Q\| g(n, p)$, where $p$ is the pair of points on the basic $1,2, \sqrt{5}$ triangle that correspond to $(P, Q)$ under similarity. Theorem 3 states that $g(n, \cdot)$ approaches 1 pointwise as $n \rightarrow \infty$.

If $P$ and $Q$ are points that are separated by Euclidean distance $|L|$, then the straight line $L$ from $P$ to $Q$ will cut across several level-n triangles, crossing the edges of the level- $n$ triangles at points $a, b, \ldots, z$. This is illustrated in Fig. 6 , in which the triangles are level- $n$, the smaller-scale tilings are not shown, and points $P, a, b, z$ and $Q$ are indicated by disks. The idea of the proof is to choose $n$ large, so $g(n, \cdot)$ is close to 1 and the shortest-path distance from each pair $(r, s)$ of consecutive points is close to the Euclidean distance. We then require $|L|$ to be large compared to $5^{n / 2}$, making the effect of the end segments $P a$ and $z Q$ negligible compared to $|L|$.

The difficulty is that the convergence $g(\cdot, n) \rightarrow 1$ is not uniform; for any $n$ there is a set of $p$ 's for which $g(n, p)$ is not close to 1 . We must control the contribution of this set, and this requires knowledge of the number of level- $n$ segments in the straight line from $P$ to $Q$.

Lemma. Let $N$ be the number of level-n segments in the straight line from $P$ to $Q$. If $|L|>(10) 5^{n / 2}$, then $5^{-(n+1) / 2}|L| \leqq N \leqq 5^{-(n-2) / 2}|L|$.

Proof. The diameter of a level- $n$ triangle is $5^{(n+1) / 2}$. This gives an upper bound on the length of each segment, and so a lower bound on the number of segments. Now, if a level- $n$ triangle intersects $L$, it lies entirely in the set of points within distance $5^{(n+1) / 2}$ of $L$. This region has area $5^{(n+1) / 2}|L|+5^{n+1} \pi$. Since each level- $n$ triangle has area $5^{n}$, there can be at most $5^{(1-n) / 2}|L|+5 \pi<5^{(2-n) / 2}|L|$ such triangles.

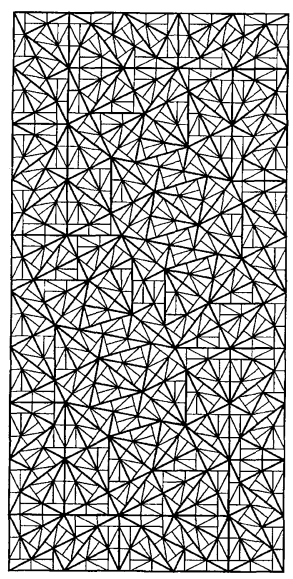

Fig. 5. Two levels of triangles 


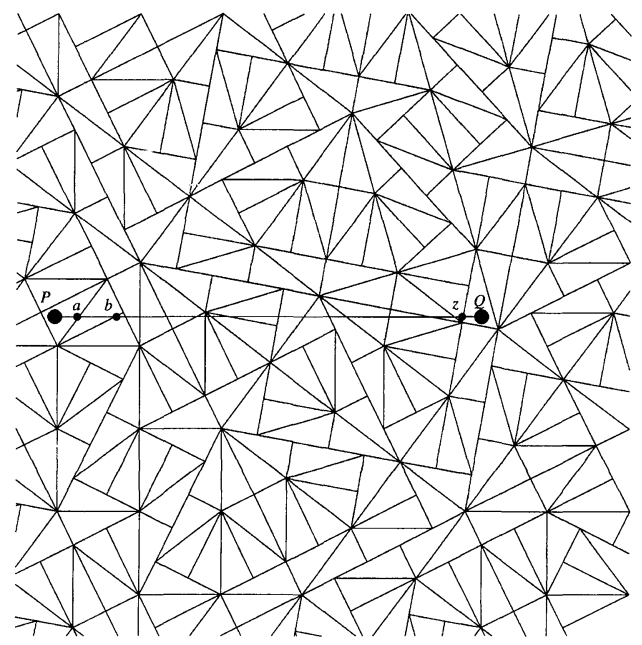

Fig. 6. Points $P, a, b, z$ and $Q$

Proof of Theorem 2. Pick $\delta=\varepsilon / 75$ and let $X^{\prime}$ be the set of pairs of points on the edges of the basic triangle that are separated by a Euclidean distance $\geqq \delta$. $X^{\prime}$ is compact, so we can find an $n$ such that $g(\cdot, n)<1+\varepsilon / 3$ on $X^{\prime}$. On $X \backslash X^{\prime}$ we have only the basic estimate $g(\cdot, n) \leqq g(\cdot, 0) \leqq 5$. Finally, pick $R>(30) 5^{(n+1) / 2} / \varepsilon$.

For any $P$ and $Q$ with $\|P-Q\|>R$, we find a path from $P$ to $Q$ as follows. Draw the straight line $L$ from $P$ to $Q$. This defines points $a, b, \ldots, z$, where $L$ crosses the edges of level- $n$ triangles, as before. Take the shortest path from $P$ to $a$, followed by the shortest path from $a$ to $b$, and so on. Some of the pairs $(r, s)$ will correspond to points in $X^{\prime}$. The total length of the resultant paths is then at most $|L|(1+\varepsilon / 3)$. Some (at most $\left.5^{(2-n) / 2}|L|\right)$ of the pairs will correspond to points in $X \backslash X^{\prime}$; the paths that result from these segments have total length at most $25 \delta|L|=|L| \varepsilon / 3$. Finally, the paths from $P$ to $a$, and from $z$ to $Q$, have total length at most $(10) 5^{(n+1) / 2}<|L| \varepsilon / 3$. Thus the total path from $P$ to $Q$ has length less than $|L|(1+\varepsilon)$.

Finally we return to our original claim (Theorem 1) about the existence of approximate circles. Given $\varepsilon>0$ there is an $N$ such that a regular $N$-gon inscribed in a circle of radius $R$ approximates it, for both length and area, to within a fraction $\varepsilon / 2$, in the sense that

$$
2 \pi R-(\text { perimeter of } N \text {-gon })<\frac{\varepsilon}{2} R, \quad \pi R^{2}-(\text { area of } N \text {-gon })<\frac{\varepsilon}{2} R^{2} .
$$

Pick a length $L$ for which the metric distance used above is Euclidean to within a fraction $\varepsilon^{2}$ and set $K=N L /(2 \pi)$. Then, for any center point and any $R>K$ we build our approximate circle as follows. Within the circle of radius $R$ around the point we inscribe a regular $\mathrm{N}$-gon in the circle, then move each of the vertices a distance $<1 / 2$ to place them on triangle edges. Connecting these points by shortest paths along triangle edges gives us an approximate $N$-gon of which we need show 
that the area and perimeter are accurate to within $\varepsilon / 2$, which would thus give us the desired approximation of the circle to within $\varepsilon$.

By assumption each approximation to a leg of the $N$-gon (we ignore corrections due to the small shifts of the vertices, as they are much smaller) has length accurate to within $\varepsilon^{2}$, and so the perimeter is also accurate to within $\varepsilon^{2}<\varepsilon / 2$. We can overestimate the error in area by assuming each approximate leg is a circular arc spanning the leg. To lowest order in the angles $\alpha$ of these arcs, the excess area is $N^{2} L^{2}(\alpha / 12 N)$ and in this notation the excess perimeter is $N L\left(\alpha^{2} / 24\right)$. From this we see that $\alpha^{2} / 24=\varepsilon^{2}$ and therefore $\alpha / 12 N=\varepsilon / \sqrt{6} N<\varepsilon / 2$.

The techniques we have used above also suffice to analyze a variety of similar problems. For example, consider the following signal-propagation problem on the vertices of the pinwheel tiling. Suppose that initially a single vertex $P$ is colored red, with all the other vertices being black. At each time step, color red the nearest neighbors of the red vertices. After a large number of steps, what will be the size and shape of the red region? In light of Theorem 2, the answer should be no surprise:

Theorem 4. Given $\varepsilon>0$, there exists an $R$ such that every vertex $Q$ with $\|P-Q\|>R$ gets colored in at most $\|P-Q\|(1+\varepsilon) / \sqrt{5}$ steps.

The problem can be restated as if the color red moved with various speeds along the different edges: $\sqrt{5}$ along hypotenuses, 1 along short legs and 2 along long legs. Since the signal cannot propagate at a speed faster than $\sqrt{5}$, the theorem shows that the red region is asymptotically a round disk of radius $\sqrt{5} n+o(n)$, where $n$ is the number of steps. (The analogous result in which one assumes the same speed on all edges is simply a restatement of Theorem 2.)

The proof of Theorem 4 is almost identical to the proof of Theorem 2 (via Theorem 3), and is essentially due to the existence of hypotenuses lying arbitrarily close to the desired direction. For the proof just assume that along hypotenuses the red color moves with speed $\sqrt{5}$ and along legs at speed 1 . (Our use of a lower speed along long legs could only raise the estimate on the number of steps.) The only subtlety is that, in order to prove the analog of Theorem 3, we must only consider subdividing by even numbers of times since only even-order subdivisions decompose hypotenuses into smaller hypotenuses and legs into smaller legs.

\section{Conclusion}

In many ways, the structure of aperiodic tilings is intermediate between periodic grids (such as cubic lattices) and stochastic grids as analyzed in percolation [Kes]. For the isoperimetric problem on pinwheel space we have shown that the stochastic features dominate. There is enough variation in the position and orientation of triangles to allow the construction of good approximate circles of arbitrary size and arbitrary center. The only essential features of the pinwheel tiling used in our proof were its hierarchical nature, which is common in the aperiodic tilings used to model quasicrystals [S-O], and its "statistical" rotational symmetry [Rad3]-the existence of edges lying close to every direction. Similar results are to be expected from any tiling with these properties. 


\section{References}

[B-R] Berend, D., Radin, C.: Are there chaotic tilings? Commun. Math. Phys. 152, 215-219 (1993)

[Gar] Gardner, M.: Extraordinary nonperiodic tiling that enriches the theory of tiles. Sci. Amer. 116-119 (January 1977)

[Kes] Kesten, H.: Percolation theory and ergodic theory of infinite particle systems. Berlin, Heidelberg, New York: Springer, 1987

[Rad1] Radin, C.: Global order from local sources. Bull. Am. Math. Soc. 25, 335-364 (1991)

[Rad2] Radin, C.: The pinwheel tilings of the plane. Ann. Math. 139, 661-702 (1994)

[Rad3] Radin, C.: Symmetry and tilings. Notices Am. Math. Soc. 42, 26-31 (1995)

[Rad4] Radin, C.: Space tilings and substitutions, Geometriae Dedicata 55, 257-264 (1995)

[Rad5] Radin, C.: Miles of tiles. Preprint, Univ. of Texas, (1994) [Obtainable from the electronic archive: mp-arc@math.utexas.edu]

[S-O] Steinhardt, P.J., Ostlund, S.: The physics of quasicrystals. Singapore: World Scientific, 1987

[Tay] Taylor, J.E.: Crystalline variational problems. Bull. Am. Math. Soc. 84, 568-588 (1978)

[Wul] Wulff, G.: Zur Frage der Geschwindigkeit des Wachstums und der Auflösung der Kriställflächen. Zeitschrift für Krystallographie und Mineralogie 34, 449-530 (1901)

Communicated by Ya.G. Sinai 
